\title{
Risk interaction of obesity, insulin resistance and hormone-sensitive lipase promoter polymorphisms (LIPE-60 C > G) in the development of fatty liver
}

\author{
Pi-Jung Hsiao ${ }^{1,6 \dagger}$, Zhih-Cherg Chen ${ }^{2 \dagger}$, Wei-Wen Hung ${ }^{1}$, Yi-Hsin Connie Yang ${ }^{3,6}$, Mei-Yueh Lee ${ }^{1}$, Jee-Fu Huang ${ }^{4,6}$ \\ and Kung-Kai Kuo $0^{5,6,7^{*}}$
}

\begin{abstract}
Background: Hormone sensitive lipase (HSL) promoter (LIPE-60 C > G) polymorphism has been found to be involved in hepatic steatosis, obesity, diabetes and dyslipidemia. The precise interactions between these risk factors and genetic susceptibility that may affect non-alcoholic fatty liver disease (NAFLD) are still not fully determined.

Methods: A cross-sectional study was conducted in 1056 men. To avoid the confounding effect of plasma glucose, the study population was classified into normal glucose tolerance (NGT, $n=729)$ and glucose intolerance $(G l, n=299)$ groups. NAFLD was diagnosed by abdominal ultrasound after ruling out any history of alcohol abuse. A multivariate regression model was used to estimate the impact of these factors on NAFLD.

Results: In the NGT group, subjects with NAFLD often have complicated metabolic abnormalities. The coexistence of NAFLD and Gl has been demonstrated to have a synergistic effect raising BMl, serum insulin and HOMA-insulin resistance (HOMA-IR). BMI and adipose-insulin resistance (Adipo-IR), but not HOMA-IR, significantly contributed to a greater risk of developing NAFLD. Serum triglyceride was significantly up-regulated in men with the (CG + GG) genotype of HSL promoter polymorphism, NAFLD and Adiopo-IR in sequence.
\end{abstract}

Conclusion: Adipo-IR, rather than HOMA-IR, appears to be a consistent insulin resistance index in the study of NAFLD. G allele of the HSL promoter polymorphism may contribute the greatest impact raising serum triglyceride in a state of glucose intolerance.

Keywords: Non-alcoholic fatty liver disease (NAFLD), Hormone-sensitive lipase (HSL), Insulin resistance, Metabolic syndrome

\section{Background}

Non-alcoholic fatty liver disease (NAFLD) is the most prevalent chronic liver disease in the world, and is growing especially quickly in Asia. It is regarded as a hepatic manifestation of metabolic syndrome (MetS) because of its close concomitance with obesity, type 2 diabetes mellitus and dyslipidemia [1,2]. Patients with NAFLD, who are at

\footnotetext{
* Correspondence: kuoksfo@yahoo.com.tw

${ }^{\dagger}$ Equal contributors

${ }^{5}$ Department of Surgery, Hepatobiliary Division, Kaohsiung Medical University

Hospital, Kaohsiung, Taiwan

${ }^{6}$ School of Medicine, College of Medicine, Kaohsiung Medical University,

Kaohsiung, Taiwan

Full list of author information is available at the end of the article
}

increased risk of atherosclerotic cardiovascular disease, are also at risk of progression to non-alcoholic steatotic hepatitis (NASH), cirrhosis or hepatocellular carcinoma [3,4]. Our previous study clearly documented a very high prevalence $(57.8 \%)$ of fatty liver, as assessed by abdominal ultrasound, in adult Taiwanese [5]. Previous study has also shown that compared to Caucasians, Asians (mostly Chinese) have a greater fat accumulation in the body and viscera for a given body mass index (BMI) [6]. Understanding the consequences and how to prevent the development of fatty liver are extremely important for Asians.

NAFLD, defined as hepatic steatosis with an intrahepatic triglyceride (TG) content $>5 \%$ of the liver volume or 
weight, develops owing to an imbalance between fatty acid (FA) input and output. Physiologically, the hepatic TG content results from a complex interaction of lipid homeostasis, including (1) fatty acid influx derived by adipose lipolysis (contributing 60\% of hepatic FA), (2) dietary fat intake from chylomicron (10-15\%), (3) de novo lipogenesis from plasma glucose (25-30\%), (4) fatty acid $\beta$-oxidation and (5) fatty acid export by esterification to secrete as a very low-density lipoprotein (VLDL) $[7,8]$. The mechanism of excess hepatic fat accumulation is attributed generally to enhanced FA delivery from adipose lipolysis and increased de novo lipogenesis in the liver itself, while $\beta$-oxidation and VLDL export play minor roles [8].

Fatty acid synthase (FAS), catalyzing the final step in FA biosynthesis, is well known to be the major determinant of the generation of hepatic FA by de novo lipogenesis. Altered FAS expression has been correlated with obesity related insulin resistance and hepatic steatosis. Therefore, circulating FAS has been suggested to be a possible surrogate marker of insulin resistance $[9,10]$. In the FA metabolism, adipose triglyceride lipase (ATGL) and hormone-sensitive lipase (HSL) are responsible for $>95 \%$ of TG hydrolysis. Both ATGL and HSL regulate the basal lipolysis, whereas only HSL determines the stimulated lipolysis. HSL, catalyzing diacylglycerol and monoacylglycerol into free fatty acids, determines the rate-limiting step to modulate complete lipolysis $[11,12]$. HSL is also engaged in the mobilization of FA from intracellular lipid stores in tissues. Insulin represents the most potent inhibitor of HSL to shut down lipolysis, and HSL expression has often been correlated with the pathogenesis of type 2 diabetes, abdominal obesity and MetS [13-15]. Insulin resistance is the pathophysiologic hallmark of the development of NAFLD. As there is a very low expression of ATGL in the liver, the activities of FAS and HSL seem to be essential for the regulation of fatty acid metabolism in the formation of NAFLD.

Genetic susceptibility to hepatic lipid accumulation is also considered important because of the evidence that approximately one-third of NAFLD occurs in subjects without the documented risk factors of obesity and insulin resistance. The Ile 1483 variant of the FAS gene was reported to have a protective effect, with a lower BMI, waist hip ratio, fasting glucose and blood pressure [16]. The well-studied promoter variant of $\operatorname{HSL}(-60 \mathrm{C}>\mathrm{G})$, exhibiting a $40 \%$ decline in promoter activity, plays a critical role in fat metabolism in some diseases in a sex-, race- and insulin-dependent manner $[17,18]$.

A combination of genetic and environmental risk factors, for example, diet, obesity or diabetes, is well known to cause the development of NAFLD $[4,19]$. However, the risk interaction and the relative impact on the development of NAFLD of individual genes and related metabolic biomarkers have not been thoroughly investigated. We designed this study to clarify the impact of metabolic abnormalities on the relationship between fatty liver and glucose intolerance. The differential impact of confounding risks for the development of NAFLD was analyzed after stratification of the fasting glucose. The results could have eventual clinical utility to help establish a practical treatment strategy for NAFLD in distinct populations with normal or abnormal glucose tolerance.

\section{Methods}

\section{Selection criteria}

Subjects were recruited from the Department of Preventive Medicine at KMUH in 2005 under the approval and supervision of the Institutional Review Board of Kaohsiung Medical University Hospital (KMUH-IRB-980323). All of the serum was obtained from the tissue bank in our hospital and de-identified from participant's names and personal characteristics. To avoid gender bias, a cross-sectional population of 1056 males was randomly enrolled within 3 months. The detailed medical history of each subject was evaluated by an experienced physician. Twenty-seven participants were excluded due to recognized dyslipidemia secondary to poorly-controlled DM, documented DM with medication, Cushing's syndrome, hypothyroidism, nephrotic syndrome, chronic liver disease, heavy alcohol use (drinking more than once weekly) or use of lipid-lowering agents. A total of 1029 male subjects were eligible for further study, and were stratified by fasting glucose into normal glucose tolerance (NGT, $<100 \mathrm{mg} / \mathrm{dl}$ ) and glucose intolerance (GI, $\geq 100 \mathrm{mg} / \mathrm{dl})$ groups.

\section{Laboratory measurements}

After overnight fasting, blood samples were collected and analyzed for serum glucose, aspartate aminotransferase (AST), alanine aminotransferase (ALT), total cholesterol, serum triglyceride, HDL-cholesterol, and LDL-cholesterol, using a multichannel autoanalyser. Serum insulin was measured using commercial radioimmunoassay kits (Diagnostic Products Corporation, Los Angeles, CA, USA). Serum non-esterified fatty acid (NEFA) was measured by colorimetry (Wako Pure Chemical Industries, Ltd, Osaka, Japan). The objectively quantitative expression of the relative hepatic insulin resistance was indicated by the homeostatic model assessment of insulin resistance (HOMA-IR index $=$ insulin $(\mu \mathrm{U} / \mathrm{mL}) \times$ glucose $(\mathrm{mmol} / \mathrm{L}) / 22.5)$. The adipose insulin resistance was expressed as the adipose insulin resistance (Adipo-IR index $=$ fasting serum NEFA $(\mu \mathrm{mol} / \mathrm{L}) \times$ fasting serum insulin $[\mu \mathrm{U} / \mathrm{mL}])[20,21]$.

\section{Search strategy}

EDTA-treated blood samples were used for DNA extraction by standard methods. The TaqMan genotyping 
assay was performed to detect the sequence of fatty acid synthase FAS polymorphisms (rs2228305 for Ile1483Val, $\underline{\text { rs2228307 }}$ for Val1888Ile) and $H S L$ promoter polymorphism (rs34845087 for C-60G, LIPE $14672 \mathrm{C}>\mathrm{G}$ ). These assays were designed according to the SNP reference data in the NCBI GenBank database. The ABI PRISM $7500^{\circ}$ sequence detection system was use to determine the sequence of the gene variants (Applied Biosystems, Roche, Taipei, Taiwan).

\section{Evaluation of fatty liver}

Sonographic diagnosis of fatty liver was performed by abdominal B-mode ultrasound (3.5 $\mathrm{MHz}$ convex transducer, Toshiba SSA-250; Toshiba, Tokyo, Japan) carried out by experienced hepatologists trained at the same institution to ensure interobserver consistency. Diagnosis of fatty liver was based on the brightness of the liver on ultrasound as compared with the kidney, vascular blurring of the hepatic vein trunk, and deep attenuation in the right hepatic lobe. The absence of fatty liver change (AFL) was defined as a normal echo texture without visible fatty change. The presence of fatty liver (FL) was defined as an increase in the fine echoes of hepatic parenchyma with impaired visualization of the intrahepatic vessels and diaphragm.

\section{Statistical analysis}

The SPSS 18.0 statistical package for Windows (SPSS Inc., Chicago, IL, USA) was used for all of the statistical analyses. Continuous variables were represented as the means \pm SD. Nonparametric tests (Kruskal-Wallis test or Mann-Whitney rank-sum test) were used when the original measurements were highly skewed. Allele frequency was estimated by direct counting, while genotype distribution with Hardy-Weinberg equilibrium was tested using the chi-square test. Two-way analysis of variance (ANOVA, $\alpha=0.05$ ) was carried out to evaluate the metabolic profiles by the interaction effects between fatty liver and glucose intolerance. Student's $t$-test with Bonferroni comparisons post hoc analysis was conducted within the NGT and GI groups. Multivariate regression analysis was further employed using fatty liver as a dependent variable, while body mass index (BMI), HOMA-IR, Adipo-IR and HSL (GG + GC vs CC) genotype were selected as independent variables based on significance in univariate analyses. To avoid multicollinearity in the regression model, serum insulin and NEFA were not included as independent variables in the multivariate regression model. Separate multiple regression analyses stratified by fasting glucose were further used to evaluate the effects of BMI, HOMA-IR, Adipo-IR, fatty liver, and HSL promoter genotypes (GG + GC vs CC) on serum TG. In addition, to compare the parameter estimates between NGT and GI, a single multiple regression model was conducted with the additional interactions of glucose intolerance vs BMI, HOMA-IR, Adipo-IR, fatty liver, and HSL promoter (GG + GC vs CC). Statistical significance was defined as a $P$ value of $<0.05$ using a two-tailed test.

\section{Results}

To standardize the de novo lipogenesis by fasting plasma glucose, our purely male population was divided into NTG $(n=729)$ and GI $(n=299)$ groups. The age of the participants ranged from 20 to 70 years, the majority (>80\%) being distributed in the range of 40-65 years. The prevalence of GI was $29.1 \%$ in our adult population. There was a high prevalence of MetS abnormalities (obesity $30.6 \%$, GI $29.1 \%$, hyperTG $42.2 \%$, low HDL-C $19.6 \%$ and hypertension 51.6\%) in subjects with NAFLD. Minor allele A of FAS (Val1483Ile) and G of FAS (Val1888Ile) polymorphism was nearly absent, with a monogenic distribution of Val1483 (99.6\%) and Val 1888 (100\%). The genetic effect of FAS was not further analyzed in the development of fatty liver. The frequency of the minor $G$ allele of the HSL promoter $(-60 \mathrm{C}>\mathrm{G}$ ) was $9.9 \%$, while the genotype frequency of CC: CG: GG was distributed as 80.8: 18.4 : $0.8 \%$ in Hardy-Weinberg equilibrium. There was no significant difference in the frequency distribution of the HSL promoter $(-60 \mathrm{C}>\mathrm{G})$ genotype between the NGT and GI groups.

As shown in Table 1, the prevalence of FL (67.2\%) in the GI group was significantly higher than in the NGT group (55.7\%) ( $p=0.001$, chi-square test). Within the NGT or GI groups, there were significantly greater metabolic abnormalities in the presence of FL. The metabolic profiles, such as BMI, serum insulin and HOMA-IR, were significantly attributed to a synergistic effect of FL and GI. However, the metabolic abnormalities in the group of NGT and FL seemed equivalent or even worse than those in the GI group without FL. The metabolic abnormalities occurred more in the presence of FL.

In the development of FL, risk analysis was conducted to compare the odds ratios of BMI, HOMA-IR, AdipoIR and HSL promoter genotypes (CC vs CG + GG) (Table 2). Analysis showed that BMI and Adipo-IR, rather than HOMA-IR and HSL promoter polymorphism, are independent risk factors for the formation of FL.

Obesity plays a central role in MetS. Our study demonstrated that the frequency of FL and the metabolic profiles of MetS were positively parallel to BMI, with the exception of GI (Table 3). The frequency of FL is greater than that of GI for a given BMI. Relevant metabolic abnormalities, including $38.4 \%$ for fatty liver, $33.4 \%$ for hypertension, $26.4 \%$ for glucose intolerance, $18.2 \%$ for hypertriglyceridemia and $10.1 \%$ for low HDL-C, existed in normal BMI subjects; this has previously been regarded as metabolic obese normal weight $(\mathrm{MONW})$. This means that hepatic steatosis is not only dependent on the BMI status, and the 
Table 1 Comparison of the metabolic abnormalities between glucose tolerance and fatty liver

\begin{tabular}{|c|c|c|c|c|c|c|c|}
\hline \multirow[t]{2}{*}{ Groups/variables } & \multicolumn{2}{|c|}{ Normal Glucose Tolerance (NGT) } & \multicolumn{2}{|c|}{ Glucose Intolerance (GI) } & \multirow{2}{*}{$\begin{array}{l}P \text { for effect } \\
\text { of FL vs AFL }\end{array}$} & \multirow{2}{*}{$\begin{array}{l}P \text { for effect } \\
\text { of Gl vs NGT }\end{array}$} & \multirow{2}{*}{$\begin{array}{l}P \text { for interaction } \\
\text { of } \mathrm{FL} \text { and } \mathrm{Gl}\end{array}$} \\
\hline & $\begin{array}{l}\text { AFL } \\
(n=323)\end{array}$ & $\begin{array}{l}\mathrm{FL} \\
(\mathrm{n}=406)\end{array}$ & $\begin{array}{l}\text { AFL } \\
(n=98)\end{array}$ & $\begin{array}{l}\mathrm{FL} \\
(\mathrm{n}=201)\end{array}$ & & & \\
\hline Age (yrs) & $45.63 \pm 8.10$ & $44.60 \pm 8.65$ & $48.68 \pm 6.89$ & $49.21 \pm 8.69$ & 0.675 & $<0.0001$ & 0.195 \\
\hline $\mathrm{BMI}\left(\mathrm{kg} / \mathrm{m}^{2}\right)$ & $23.16 \pm 2.51$ & $25.64 \pm 3.06^{*}$ & $22.79 \pm 3.31$ & $26.16 \pm 3.03^{*}$ & $<0.0001$ & 0.716 & 0.035 \\
\hline Serum insulin $(\mu \mathrm{IU} / \mathrm{ml})$ & $2.22 \pm 2.47$ & $4.28 \pm 4.48^{*}$ & $3.77 \pm 6.73$ & $7.74 \pm 12.00^{*}$ & $<0.0001$ & $<0.0001$ & 0.044 \\
\hline NEFA ( $\mu \mathrm{mol} / \mathrm{L})$ & $509.81 \pm 192.78$ & $575.03 \pm 227.93^{*}$ & $589.47 \pm 230.07$ & $646.75 \pm 264.183$ & $<0.0001$ & $<0.0001$ & 0.811 \\
\hline HOMA-IR & $0.48 \pm 0.53$ & $0.94 \pm 0.99^{*}$ & $1.28 \pm 2.89$ & $2.47 \pm 4.49^{*}$ & $<0.0001$ & $<0.0001$ & 0.027 \\
\hline Adipo-IR & $1.11 \pm 1.22$ & $2.57 \pm 3.95^{*}$ & $2.10 \pm 3.86$ & $4.50 \pm 5.24^{*}$ & $<0.0001$ & $<0.0001$ & 0.083 \\
\hline $\mathrm{SBP}(\mathrm{mmHg})$ & $123.5 \pm 15.3$ & $126.2 \pm 15.1^{*}$ & $126.86 \pm 16.86$ & $130.44 \pm 18.20$ & 0.007 & 0.001 & 0.701 \\
\hline $\mathrm{DBP}(\mathrm{mmHg})$ & $76.2 \pm 10.9$ & $79.1 \pm 10.6^{*}$ & $78.55 \pm 11.49$ & $82.45 \pm 11.83^{*}$ & $<0.0001$ & $<0.0001$ & 0.530 \\
\hline AST (IU/L) & $27.20 \pm 11.81$ & $29.97 \pm 11.41^{*}$ & $28.77 \pm 16.31$ & $34.22 \pm 18.71^{*}$ & $<0.0001$ & 0.004 & 0.163 \\
\hline ALT (IU/L) & $25.28 \pm 19.78$ & $34.35 \pm 20.64^{*}$ & $25.94 \pm 20.91$ & $40.70 \pm 33.01^{*}$ & $<0.0001$ & 0.037 & 0.090 \\
\hline Cholesterol (mg/dl) & $175.13 \pm 31.00$ & $184.24 \pm 32.65^{*}$ & $173.98 \pm 28.97$ & $188.17 \pm 34.57^{*}$ & $<0.0001$ & 0.548 & 0.274 \\
\hline Triglyceride (mg/dl) & $104.78 \pm 58.24$ & $158.29 \pm 115.18^{*}$ & $129.99 \pm 111.28$ & $163.97 \pm 102.82^{*}$ & $<0.0001$ & 0.028 & 0.165 \\
\hline $\mathrm{HDL}-\mathrm{C}(\mathrm{mg} / \mathrm{dl})$ & $53.75 \pm 12.87$ & $49.12 \pm 11.55^{*}$ & $50.09 \pm 10.67$ & $48.30 \pm 10.68$ & 0.002 & 0.034 & 0.176 \\
\hline LDL-C (mg/dl) & $115.27 \pm 31.50$ & $130.27 \pm 32.97^{*}$ & $117.23 \pm 28.96$ & $129.08 \pm 33.12^{*}$ & $<0.0001$ & 0.895 & 0.584 \\
\hline
\end{tabular}

${ }^{*} \mathrm{P}<0.025=0.05 / 2$ (Bonferroni test, two sample comparison within the normal glucose tolerance and glucose intolerance groups).

AFL indicates absence of fatty liver, FL indicates fatty liver. BMI indicates body mass index. SBP and DBP indicate systolic and diastolic blood pressure. NEFA indicates non-esterified fatty acid.

threshold for hepatic fat accumulation essentially differs greatly between individuals.

Power calculation was more than $80 \%$ in the whole population when the pooled standard deviation of TG is estimated around $90 \mathrm{mg} / \mathrm{dl}$. In the NGT group, the presence of FL followed by high Adipo-IR and BMI significantly contributed to a raised serum TG (Table 4). However, the effect of BMI on serum TG disappeared in the GI group. Conversely, the HSL promoter genotype (CG + GG vs CC) demonstrated a significant strong impact elevating serum TG in the GI but not the NGT group. Comparison of the parameter estimates of the impact on serum TG, indicated by interactions, showed

Table 2 Logistic regression using fatty liver as a dependent variable in normal glucose tolerance and glucose intolerance groups

\begin{tabular}{lll}
\hline Independent variables & Odds ratio & $P$ value \\
\hline Normal glucose tolerance & $1.31(1.22-1.41)$ & $<0.0001$ \\
BMI & $0.98(0.55-1.77)$ & 0.948 \\
HOMA-IR & $1.36(1.06-1.75)$ & 0.016 \\
Adipo-IR & $1.41(0.71-2.16)$ & 0.122 \\
HSL promoter genotype (CC vs CG + GG) & \\
Glucose intolerance & $1.50(1.32-1.69)$ & $<0.0001$ \\
BMI & $0.85(0.64-1.13)$ & 0.258 \\
HOMA-IR & $1.41(1.11-1.78)$ & 0.005 \\
Adipo-IR & $1.03(0.49-2.18)$ & 0.940 \\
HSL promoter genotype (CC vs CG + GG)
\end{tabular}

a significant difference in the HSL promoter (GG + GC vs CC) $(p=0.005)$ between the NGT and the GI groups. Therefore, it means that the TG level is very dependent upon the status of the subject's glucose intolerance or not.

\section{Discussion}

Our study demonstrated that BMI and Adipo-IR significantly contributed to FL formation. Men's BMI is reported to predict approximately $93 \%$ of the variance linked to obesity-related adipocyte size, fat cell mass and fat distribution [22]. Accordingly, BMI could be used as a clinically practical parameter in males to represent the obesity-related fat metabolism. There is substantial evidence that obesity is related to excess fat cell mass leading to increased basal lipolysis [11]. Insulin resistance is well-documented as the hallmark of MetS, but occurs

Table 3 Prevalence of fatty liver and indexes of metabolic syndrome by BMI status

\begin{tabular}{lllll}
\hline BMI status $\left(\mathbf{k g} / \mathbf{m}^{\mathbf{2}}\right)$ & $\begin{array}{l}<\mathbf{2 4} \\
(\mathbf{n = 4 3 4})\end{array}$ & $\begin{array}{l}\mathbf{2 4}-\mathbf{2 6 . 9} \\
\mathbf{( n = 3 7 9 )}\end{array}$ & $\begin{array}{l}\geq \mathbf{2 7} \\
\mathbf{( n = 2 1 6 )}\end{array}$ & $\mathbf{P}$ \\
\hline Fatty liver & $38.4 \%$ & $67.3 \%$ & $86.1 \%$ & $<0.0001$ \\
Hypertension & $33.4 \%$ & $53.0 \%$ & $57.9 \%$ & $<0.0001$ \\
Glucose intolerance & $26.4 \%$ & $29.2 \%$ & $34.9 \%$ & 0.086 \\
Hypertriglyceridemia & $18.2 \%$ & $37.4 \%$ & $49.3 \%$ & $<0.0001$ \\
HDL $<40 \mathrm{mg} / \mathrm{dl}$ & $10.1 \%$ & $22.6 \%$ & $24.6 \%$ & $<0.0001$ \\
\hline
\end{tabular}

Hypertension is defined as systolic $\mathrm{BP} \geq 130$ or diastolic $\mathrm{BP} \geq 80 \mathrm{mmHg}$. Glucose intolerance is defined as fasting glucose $\geq 100 \mathrm{mg} / \mathrm{dl}$.

Hypertriglyceridemia is defined as fasting triglyceride $\geq 150 \mathrm{mg} / \mathrm{dl}$. 


\begin{tabular}{|c|c|c|c|c|}
\hline \multirow[t]{2}{*}{ Independent variables } & \multirow{2}{*}{$\begin{array}{l}\text { Parameter } \\
\text { estimates (B) }\end{array}$} & \multicolumn{2}{|c|}{ 95\% Confidence interval } & \multirow[t]{2}{*}{$P$ value } \\
\hline & & Lower bound & Upper bound & \\
\hline \multicolumn{5}{|l|}{ Normal glucose tolerance } \\
\hline BMI & 4.30 & 2.41 & 6.20 & $<0.0001$ \\
\hline HOMA-IR & -31.06 & -41.11 & -21.01 & $<0.0001$ \\
\hline Adipo-IR & 17.04 & 14.35 & 19.74 & $<0.0001$ \\
\hline FL vs AFL & 27.50 & 16.41 & 38.58 & $<0.0001$ \\
\hline HSL promoter genotype (CG + GG vs CC) & -3.05 & -16.14 & 10.04 & 0.648 \\
\hline \multicolumn{5}{|l|}{ Glucose intolerance } \\
\hline BMl & -0.32 & -3.82 & 3.82 & 0.860 \\
\hline HOMA-IR & -11.01 & -16.26 & -5.76 & $<0.0001$ \\
\hline Adipo-IR & 11.50 & 7.04 & 15.96 & $<0.0001$ \\
\hline FL vs AFL & 29.40 & 3.74 & 55.05 & 0.025 \\
\hline HSL promoter genotype (CG + GG vs CC) & 34.10 & 9.16 & 60.04 & 0.010 \\
\hline
\end{tabular}

FL indicates fatty liver, AFL indicates absence of fatty liver.

with a differential response intensity of target tissues such as adipose, liver and muscle tissues to a given insulin level [23]. The Adipo-IR index is a simple way of measuring the inhibiting response of lipolysis by insulin in adipose tissue. Our results rationally suggest that people with a higher BMI usually have a greater fat cell mass, increased basal lipolysis leading to higher NEFA and greater insulin resistance (represented by Adipo-IR), which will result in hepatic FFA overflow and FL formation. The genetic effect of the HSL promoter (CC vs CG + GG) in relevant lipolysis and FL formation may be overcome by the greater impact of obesity-related lipolysis and insulin resistance of adipose (Adipo-IR). This finding is compatible with the fact that increased plasma FFA from adipose lipolysis is the major provider of lipids in the steatotic liver $[8,20,24]$.

NAFLD has been regarded as the hepatic manifestation of MetS. However, debate continues regarding the progression of MetS to NAFLD or vice versa $[25,26]$. NAFLD has been reported to be a better independent risk factor for cardiovascular disease than MetS in some studies [3,27]. Liver fat is an independent determinant of the hepatic glucose output, which is sensitive to insulin. The liver is the primary site regulating $50-70 \%$ of insulin clearance [28]. Increased hepatic steatosis is associated with impaired insulin clearance and hepatic insulin resistance, which results in plasma glucose elevation, compensatory hyperinsulinemia and progression to type 2 DM. Hepatic insulin resistance also accelerates VLDL secretion, contributing to hypertriglyceridemia and lowHDL cholesterol concentration. Thus, hyperinsulinemia is regarded to be a consequence rather than a cause of NAFLD. Furthermore, hyperinsulinemia, stimulating the sympathetic tone and enhancing renal sodium reabsorption, contributes to hypertension [28,29]. HOMA-IR, an indirect estimate of insulin resistance, is usually used to represent the inhibition of hepatic glucose output by insulin, which is maintained by a balance between the liver and $\beta$-cells [20]. As suggested by Kotronen et al., the HOMA-IR index, which overestimates the insulin resistance in subjects with NAFLD, is more likely to reflect impaired insulin clearance and secretion than insulin sensitivity [29]. Our results revealed an inconsistent effect of HOMA-IR, whereas Adipo-IR showed a consistent trend in regulating NAFLD formation or serum TG. This highly suggests Adipo-IR as an index of insulin sensitivity in NAFLD or MetS research. In our study, subjects with FL and NGT complicated more metabolic abnormalities than subjects with AFL and GI. The coexistence of FL and GI exerts a significant synergistic effect raising BMI, serum insulin and HOMA-IR. This indicates that FL may develop earlier in the progression of MetS and the coexistence of FL and GI may magnify obesity and insulin resistance.

No matter how MetS is defined, visceral obesity is an obligatory component of MetS based on the recommendations of the International Diabetes Federation consensus [30]. However, there is still no convenient, practical worldwide indicator to define visceral obesity. As our study has shown, metabolic abnormalities are considerably prevalent in a MONW population. Visceral obesity is not easily defined by BMI alone since the threshold for hepatic lipid accumulation (a critical visceral adiposity) differs individually [4]. The liver is a pivotal organ to regulate the homeostasis of fat and glucose. It also plays a central role linking the interaction of obesity, GI, 
hypertension and dyslipidemia. Previous evidence has shown that hepatic steatosis rather than extrahepatic adiposity is more correlated with insulin resistance, especially in normal-weight non-diabetic subjects. NAFLD is an early manifestation of MetS and its severity is positively parallel to the degree of obesity. Therefore, hepatic steatosis may be the earliest sign in the pathogenesis of MetS and may be a better marker of visceral obesity for defining MetS, especially in a MONW population $[4,26,31]$. Compared with the gold standard of liver biopsy to diagnose FL, abdominal ultrasound is a noninvasive, convenient and accurate tool with high sensitivity and specificity. Therefore, we propose that a steatotic liver evaluated by ultrasound is a more sensitive indicator than BMI for defining visceral obesity.

Facing an increased FA influx and de novo lipogenesis, the hepatic FA pool is regulated by $\beta$-oxidation, with biosynthesis of TG for secretion as VLDL-C particles or storage as intrahepatic lipid. Current evidence suggests that hepatic TG synthesis and VLDL-TG secretion protect against lipotoxicity by buffering hepatic FFA influx [8]. Fasting serum TG is carried predominantly in the particles of VLDL secreted from the liver, which is inhibited by insulin. In subjects without FL, nearly $70 \%$ of FA incorporated into VLDL-TG is derived from plasma FA sources, and the rest originates from hepatic de novo lipogenesis and lipolysis of intrahepatic lipids. The VLDL-TG secretion rate is greater in subjects with FL than those without FL [4,7]. Our results demonstrated that the impact of increased circulating TG is significantly regulated by the presence of FL, Adipo-IR and BMI in sequence. This is compatible with the reported fact that a higher $\mathrm{BMI}$, greater insulin resistance to adipose (Adipo-IR) and more liver fat is compensated with higher secretion of VLDL-TG $[4,7,8,30]$. Therefore, the presence of FL essentially could result in dyslipidemia and related atherosclerosis. Our results demonstrated a differential intensity of HOMA-IR inhibition of VLDL-TG secretion in the NGT and GI groups. In the GI state, it still demonstrated an inhibiting impact on VLDL-TG secretion coexistent with the impaired hepatic output in a given HOMA-IR, which implies differential insulin sensitivity to regulate fat and glucose metabolism in the liver, such as by inhibiting VLDL-TG secretion and hepatic glucose output. However, greater insulin resistance has been shown to lead to greater VLDL-TG secretion and higher serum TG [12-14]. Thus our variable $\mathrm{TG}$ regulation responses when using HOMA-IR as an insulin resistance index suggest the need for a more appropriate index to represent insulin resistance for glucose or fatty acid metabolism. Adipo-IR, representing the circulating FFA influx relative to insulin, can be regarded as a good indicator of insulin resistance in studies of TG metabolism and NAFLD.
There are many reports in the literature investigating C-60G gene polymorphism in the HSL promoter. The Ely study showed a gender specific effect on insulin and lipid levels in-60G carriers. Men carrying the -60G allele had significantly lower fasting NEFA and LDLcholesterol than non-carriers [18]. Ordovas et al. reported that male carriers of the $-60 \mathrm{G}$ allele who were not alcohol drinkers had higher glucose levels than noncarriers [32]. In addition, the C-60G polymorphism is associated with increased waist circumference in lean subjects [33]. The interaction between body fat mass and physical activity is closely associated with the C-60G polymorphism in male carriers. The Quebec Family study showed that men who were $G$ allele carriers were less likely to lose adiposity by physical activity than noncarriers [34]. Talmud et al. found no significant difference in fasting lipid, glucose, BMI, waist/hip ration or blood pressure between $C$ and $G$ allele carriers but the $G$ allele carriers had significant lower HOMA index in healthy young men [35]. Taken together, these previous reports reveal that HSL promoter polymorphisms play a critical role in the regulation of fat and glucose metabolism and are also highly correlated with insulin resistance. The apparent discrepancies between these studies, however, are hard to rationally explain via pathophysiologic mechanisms. To avoid confounding effects, multivariate regression analysis was carried out focusing only on male gender stratified by fasting glucose so insulin resistance is clearly defined. Our results demonstrated different impacts on serum TG by insulin resistance, BMI and the HSL promoter genotype after stratification by serum glucose. Since serum insulin, HOMA-IR and BMI were significantly attributable to a synergistic effect of glucose intolerance and FL, it is necessary to compare the interaction of these confounding factors together on serum TG. We observed no difference in anthropometric or metabolic parameters and related insulin resistance indexes between genotype $(\mathrm{CG}+\mathrm{GG})$ and $(\mathrm{CC})$ carriers in the NTG group, except for significantly higher serum TG levels found in carriers of the $G$ allele in the GI group (Additional file 1: Table S1 and S2).

Recent evidence has shown that the accumulation of diacylglycerol in the liver might activate protein kinase $\mathrm{C}$, induce insulin resistance and increase VLDL-TG secretion. HSL is involved in a rate-limiting step to catalyze diacylglycerol and monoacylglycerol into free fatty acids, so HSL activity determines the tissue content of diacylglycerol and the degree of insulin resistance $[20,26]$. Reid et al. found that hepatic overexpression of HSL promotes $\beta$-oxidation of the fatty acid to ameliorate steatosis [36]. Taken together, HSL activity has a critical role in the development of NAFLD. The G allele of the HSL promoter (C-60G) exhibited a $40 \%$ decline in promoter activity, which may theoretically cause greater 
accumulation of diacylglycerol in the liver. This could lead to greater insulin resistance, less $\beta$-oxidation and more VLDL-TG secretion. The HSL activity is negatively regulated by insulin and up-regulated by glucose. There are multiple prominent cis-regulatory elements, including the insulin response element (IRE), glucose response element (GRE) and fat specific element (FSE), along with other transcriptional factor binding elements, residing in the proximal promoter regions of the human HSL gene. All these cis-acting elements act as critical transcriptional regulators for promoter activity [37,38]. A literature review by Ling et al. pointed out that the epigenetic effect of DNA methylation always occurs in CG dinucleotides and is modified by environmental exposure to nutrients in complex multifactorial diseases such as type $2 \mathrm{DM}$. The relative ratio of insulin, glucose and fat levels may act on the cis-regulating elements to modulate promoter function and transcriptional initiation [39]. Our results showed a genetic effect of the HSL promoter (C-60G) regulating serum TG only in the GI group and not in the NGT group. It is rational to speculate that the $\mathrm{G}$ allele of the HSL promoter may express a differential susceptibility to the complex interaction between serum insulin, glucose and fat to determine lipolysis, such as lowering lipolytic activity only under a dependable range of insulin resistance. Because this is a cross-sectional study, further prospective and large-scaled study is suggested to verify this preliminary observation.

\section{Conclusion}

The risk interaction between genetic susceptibility, obesity and insulin resistance contribute different impacts on the development of NAFLD. Adipo-IR, rather than HOMA-IR, is proposed as a consistent insulin resistance index for the study of NAFLD. BMI and Adipo-IR were independent risk factors for the formation of FL. In a GI state, HSL promoter $(\mathrm{CG}+\mathrm{GG})$ contributed the greatest impact on raising serum TG, followed by FL and AdipoIR in sequence.

\section{Additional file}

Additional file 1: Table S1. Comparison of biochemistry between genotypes $(\mathrm{CG}+\mathrm{GG})$ and $(\mathrm{CC})$ of the hormone sensitive lipase (HSL) promoter in the normal glucose tolerance group $(n=729)$. S2. Comparison of biochemistry between genotypes ( $C G+G G)$ and $(C C)$ of the hormone sensitive lipase (HSL) promoter in the glucose intolerance group ( $\mathrm{n}=299)$

\section{Abbreviations}

NAFLD: Non-alcoholic fatty liver disease; MetS: Metabolic syndrome; NASH: Non-alcoholic steatotic hepatitis; BMI: Body mass index;

TG: Triglyceride; FA: Fatty acid; VLDL: Very low-density lipoprotein; FAS: Fatty acid synthase; ATGL: Adipose triglyceride lipase; HSL: Hormone-sensitive lipase; NGT: Normal glucose tolerance; Gl: Glucose intolerance; AST: Aspartate aminotransferase; ALT: Alanine aminotransferase; NEFA: Non-esterified fatty acid; HOMA-IR: Homeostatic model assessment of insulin resistance; Adipo-
IR: Adipose insulin resistance; FL: Fatty liver; AFL: Absent fatty liver; MONW: Metabolic obese normal weight; IRE: Insulin response element; GRE: Glucose response element; FSE: Fat specific element.

\section{Competing interests}

All of the authors contributed substantially to this study. All of the authors fully declare that there is no conflict of interest prejudicing the text of this article.

\section{Authors' contributions}

$\mathrm{PJH}$ involved research design, quality control of the genetic experiments, and writing the manuscript. ZCC designed the research. WWH searched the literature and analyzed data. YHCY contributed to using analytic tools for statistical analysis and involved the statistical analysis. MYL collected data for analysis. JFH contributed on patients enrollment. KKK supervised the project, edit and revise the manuscript. All authors read and approved the final manuscript.

\section{Acknowledgements}

This study was sponsored by grants from the cooperative research program at Chi-Mei Medical Center and Kaohsiung Medical University, CMFHR9647. We are sincerely grateful to Shu-Ting Lee for technical assistance.

\section{Author details}

${ }^{1}$ Department of Internal Medicine, Division of Endocrinology and Metabolism, Kaohsiung Medical University Hospital, Kaohsiung, Taiwan. ²Department of Medical Research, Section of Internal Medicine, Chi-Mei Medical Center, Tainan, Taiwan. ${ }^{3}$ Biostatistics, in the Statistical Analysis Laboratory, Kaohsiung Medical University Hospital, School of Pharmacy, Kaohsiung, Taiwan. ${ }^{4}$ Department of Internal Medicine, Hepatobiliary Division, Kaohsiung Medical University Hospital, Kaohsiung, Taiwan. ${ }^{5}$ Department of Surgery, Hepatobiliary Division, Kaohsiung Medical University Hospital, Kaohsiung, Taiwan. ${ }^{6}$ School of Medicine, College of Medicine, Kaohsiung Medical University, Kaohsiung, Taiwan. ${ }^{7}$ Department of Surgery, Division of Hepato-Bilio-Pancreatic Surgery, Kaohsiung Medical University, Hospital 100 Tzyou 1st Rd, Kaohsiung 807, Taiwan.

Received: 9 September 2012 Accepted: 9 May 2013 Published: 20 May 2013

\section{References}

1. Fan JG, Farrell GC: Epidemiology of non-alcoholic fatty liver disease in China. J Hepatol 2009, 50(1):204-210.

2. Fan JG, Saibara T, Chitturi S, Kim BI, Sung JJ, Chutaputti A: What are the risk factors and settings for non-alcoholic fatty liver disease in Asia-Pacific? J Gastroenterol Hepatol 2007, 22(6):794-800.

3. Ritchie SA, Connell JM: The link between abdominal obesity, metabolic syndrome and cardiovascular disease. Nutr Metab Cardiovasc Dis 2007, 17(4):319-326

4. Larter CZ, Chitturi S, Heydet D, Farrell GC: A fresh look at NASH pathogenesis. Part 1: the metabolic movers. J Gastroenterol Hepatol 2010, 25(4):672-690.

5. Hsiao PJ, Kuo KK, Shin SJ, Yang YH, Lin WY, Yang JF, Chiu CC, Chuang WL, Tsai TR, Yu ML: Significant correlations between severe fatty liver and risk factors for metabolic syndrome. J Gastroenterol Hepatol 2007, 22(12):2118-2123.

6. Wang J, Thornton JC, Russell M, Burastero S, Heymsfield S, Pierson RN Jr: Asians have lower body mass index (BMI) but higher percent body fat than do whites: comparisons of anthropometric measurements. Am J Clin Nutr 1994, 60(1):23-28.

7. Fabbrini E, Sullivan S, Klein S: Obesity and nonalcoholic fatty liver disease: biochemical, metabolic, and clinical implications. Hepatology 2010, 51(2):679-689.

8. Postic C, Girard J: Contribution of de novo fatty acid synthesis to hepatic steatosis and insulin resistance: lessons from genetically engineered mice. J Clin Invest 2008, 118(3):829-838.

9. Fernandez-Real JM, Menendez JA, Moreno-Navarrete JM, Bluher M, VazquezMartin A, Vazquez MJ, Ortega F, Dieguez C, Fruhbeck G, Ricart W, et al: Extracellular fatty acid synthase: a possible surrogate biomarker of insulin resistance. Diabetes 2010, 59(6):1506-1511.

10. Dorn C, Riener MO, Kirovski G, Saugspier M, Steib K, Weiss TS, Gabele E, Kristiansen G, Hartmann A, Hellerbrand C: Expression of fatty acid 
synthase in nonalcoholic fatty liver disease. Int J Clin Exp Pathol 2010, 3(5):505-514.

11. Carmen GY, Victor SM: Signalling mechanisms regulating lipolysis. Cell Signal 2006, 18(4):401-408.

12. Jaworski K, Sarkadi-Nagy E, Duncan RE, Ahmadian M, Sul HS: Regulation of triglyceride metabolism. IV. Hormonal regulation of lipolysis in adipose tissue. Am J Physiol Gastrointest Liver Physiol 2007, 293(1):G1-G4.

13. Avramoglu RK, Basciano H, Adeli K: Lipid and lipoprotein dysregulation in insulin resistant states. Clin Chim Acta 2006, 368(1-2):1-19.

14. Girard J, Lafontan M: Impact of visceral adipose tissue on liver metabolism and insulin resistance. Part II: visceral adipose tissue production and liver metabolism. Diabetes Metab 2008, 34(5):439-445.

15. Jocken JW, Langin D, Smit E, Saris WH, Valle C, Hul GB, Holm C, Arner P, Blaak EE: Adipose triglyceride lipase and hormone-sensitive lipase protein expression is decreased in the obese insulin-resistant state. J Clin Endocrinol Metab 2007, 92(6):2292-2299.

16. Moreno-Navarrete JM, Botas P, Valdes S, Ortega FJ, Delgado E, VazquezMartin A, Bassols J, Pardo G, Ricart W, Menendez JA, et al: Val1483lle in FASN gene is linked to central obesity and insulin sensitivity in adult white men. Obesity (Silver Spring) 2009, 17(9):1755-1761.

17. Talmud PJ, Palmen J, Walker M: Identification of genetic variation in the human hormone-sensitive lipase gene and $5^{\prime}$ sequences: homology of $5^{\prime}$ sequences with mouse promoter and identification of potential regulatory elements. Biochem Biophys Res Commun 1998, 252(3):661-668.

18. Talmud PJ, Palmen J, Luan J, Flavell D, Byrne CD, Waterworth DM, Wareham $\mathrm{NJ}$ : Variation in the promoter of the human hormone sensitive lipase gene shows gender specific effects on insulin and lipid levels: results from the Ely study. Biochim Biophys Acta 2001, 1537(3):239-244.

19. Bugianesi E, McCullough AJ, Marchesini G: Insulin resistance: a metabolic pathway to chronic liver disease. Hepatology 2005, 42(5):987-1000.

20. Neuschwander-Tetri BA: Hepatic lipotoxicity and the pathogenesis of nonalcoholic steatohepatitis: the central role of nontriglyceride fatty acid metabolites. Hepatology 2010, 52(2):774-788.

21. Gastaldelli A, Harrison SA, Belfort-Aguilar R, Hardies LJ, Balas B, Schenker S, Cusi $\mathrm{K}$ : Importance of changes in adipose tissue insulin resistance to histological response during thiazolidinedione treatment of patients with nonalcoholic steatohepatitis. Hepatology 2009, 50(4):1087-1093.

22. Mundi MS, Karpyak MV, Koutsari C, Votruba SB, O'Brien PC, Jensen MD: Body fat distribution, adipocyte size, and metabolic characteristics of nondiabetic adults. J Clin Endocrinol Metab 2010, 95(1):67-73.

23. Kovacs P, Stumvoll M: Fatty acids and insulin resistance in muscle and liver. Best Pract Res Clin Endocrinol Metab 2005, 19(4):625-635.

24. Ferre $P$, Foufelle F: Hepatic steatosis: a role for de novo lipogenesis and the transcription factor SREBP-1c. Diabetes Obes Metab 2010, 12(Suppl 2):83-92.

25. Bruce KD, Hanson MA: The developmental origins, mechanisms, and implications of metabolic syndrome. J Nutr 2010, 140(3):648-652.

26. Vanni E, Bugianesi E, Kotronen A, De Minicis S, Yki-Jarvinen H, SvegliatiBaroni G: From the metabolic syndrome to NAFLD or vice versa? Dig Liver Dis 2010, 42(5):320-330.

27. Alkhouri N, Tamimi TA, Yerian L, Lopez R, Zein NN, Feldstein AE: The inflamed liver and atherosclerosis: a link between histologic severity of nonalcoholic fatty liver disease and increased cardiovascular risk. Dig Dis Sci 2010, 55(9):2644-2650.

28. Kotronen A, Vehkavaara S, Seppala-Lindroos A, Bergholm R, Yki-Jarvinen H: Effect of liver fat on insulin clearance. Am J Physiol Endocrinol Metab 2007, 293(6):E1709-E1715.

29. Kotronen A, Juurinen L, Tiikkainen M, Vehkavaara S, Yki-Jarvinen H: Increased liver fat, impaired insulin clearance, and hepatic and adipose tissue insulin resistance in type 2 diabetes. Gastroenterology 2008, 135(1): $122-130$.

30. Scaglione R, Di Chiara T, Cariello T, Licata G: Visceral obesity and metabolic syndrome: two faces of the same medal? Intern Emerg Med 2010, 5(2): $111-119$.

31. Wasada T, Kasahara T, Wada J, Jimba S, Fujimaki R, Nakagami T, Iwamoto Y: Hepatic steatosis rather than visceral adiposity is more closely associated with insulin resistance in the early stage of obesity. Metabolism 2008, 57(7):980-985.

32. Qi L, Shen H, Larson I, Barnard JR, Schaefer EJ, Ordovas JM: Genetic variation at the hormone sensitive lipase: gender-specific association with plasma lipid and glucose concentrations. Clin Genet 2004, 65(2):93-100.

33. Carlsson E, Johansson LE, Strom K, Hoffstedt J, Groop L, Holm C, Ridderstrale M: The hormone-sensitive lipase C-60G promoter polymorphism is associated with increased waist circumference in normal-weight subjects. Int J Obes (Lond) 2006, 30(9):1442-1448.

34. Garenc C, Vohl MC, Bouchard C, Perusse L: LIPE C-60G influences the effects of physical activity on body fat and plasma lipid concentrations: the quebec family study. Hum Genomics 2009, 3(2):157-168.

35. Talmud PJ, Palmen J, Nicaud V, Tiret L: Association of the hormone sensitive lipase $-60 \mathrm{C}>\mathrm{G}$ variant with fasting insulin levels in healthy young men. Nutr Metab Cardiovasc Dis 2002, 12(4):173-177.

36. Reid BN, Ables GP, Otlivanchik OA, Schoiswohl G, Zechner R, Blaner WS, Goldberg IJ, Schwabe RF, Chua SC Jr, Huang LS: Hepatic overexpression of hormone-sensitive lipase and adipose triglyceride lipase promotes fatty acid oxidation, stimulates direct release of free fatty acids, and ameliorates steatosis. J Biol Chem 2008, 283(19):13087-13099.

37. Lafontan M, Langin D: Lipolysis and lipid mobilization in human adipose tissue. Prog Lipid Res 2009, 48(5):275-297.

38. Lampidonis AD, Stravopodis DJ, Voutsinas GE, Messini-Nikolaki N, Stefos GC, Margaritis LH, Argyrokastritis A, Bizelis I, Rogdakis E: Cloning and functional characterization of the $5^{\prime}$ regulatory region of ovine Hormone Sensitive Lipase (HSL) gene. Gene 2008, 427(1-2):65-79.

39. Ling C, Groop L: Epigenetics: a molecular link between environmental factors and type 2 diabetes. Diabetes 2009, 58(12):2718-2725.

\section{doi:10.1186/1471-2350-14-54}

Cite this article as: Hsiao et al:: Risk interaction of obesity, insulin resistance and hormone-sensitive lipase promoter polymorphisms (LIPE$60 \mathrm{C}>\mathrm{G}$ ) in the development of fatty liver. BMC Medical Genetics 2013 $14: 54$.

\section{Submit your next manuscript to BioMed Central and take full advantage of:}

- Convenient online submission

- Thorough peer review

- No space constraints or color figure charges

- Immediate publication on acceptance

- Inclusion in PubMed, CAS, Scopus and Google Scholar

- Research which is freely available for redistribution 